Signal \& Image Processing: An International Journal (SIPIJ) Vol.9, No.6, December 2018

\title{
EFFECTIVE INTEREST REGION ESTIMATION MODEL TO REPRESENT CORNERS FOR IMAGE
}

\author{
Naurin Afrin and Wei Lai \\ Department of Computer Science and Software Engineering, Swinburne University of \\ Technology, Australia
}

\begin{abstract}
One of the most important steps to describe local features is to estimate the interest region around the feature location to achieve the invariance against different image transformation. The pixels inside the interest region are used to build the descriptor, to represent a feature. Estimating the interest region around a corner location is a fundamental step to describe the corner feature. But the process is challenging under different image conditions. Most of the corner detectors derive appropriate scales to estimate the region to build descriptors. In our approach, we have proposed a new local maxima-based interest region detection method. This region estimation method can be used to build descriptors to represent corners. We have performed a comparative analysis to match the feature points using recent corner detectors and the result shows that our method achieves better precision and recall results than existing methods.
\end{abstract}

\section{KEYWORDS}

Corner, Interest Region, Descriptor, Contour-based Corner, Curvature Maxima

\section{INTRODUCTION}

Feature extraction is the most important step of the computer vision applications such as image matching, object recognition. A local feature based method consists of four stages- feature detection, interest region estimation, feature description and finally the feature matching stage [17]. To detect a feature, feature detectors takes the image as input and outputs only the feature location with no other information. The neighbourhood pixels around the feature locations forms a region that is called the interest region based on the content. The interest region is estimated to describe the feature and then feature descriptors encode relevant information of the features as a vector which acts as numerical fingerprint to match corresponding features. If the contents of the corresponding interest regions are not same, then it is almost impossible to match the descriptors of the corresponding feature locations of two images. This property is known as scale invariance which can be achieved if the features between two images match under two different scales. An effective interest region estimation method defines the neighbourhood pixels around the corner location. These pixel information later used to build scale-invariant descriptors.

Interest regions can be estimated in two ways- 1) at the time of feature detection and 2) at the time of feature description. Most of the local feature based method estimates the region at the time of feature detection using a3-dimensional scale-space [18]. Scale-space is computationally very expensive because it uses a large amount of data. It also suffers from the localization error of the actual feature location. Prominent feature like corners, estimates the region during both detection and descriptor stage. Among the two types of corner detectors, intensity-based corner detectors are derivative-based and use the scale-space to estimate region $[4,5]$. But this detectors

DOI : 10.5121/sipij.2018.9603 
Signal \& Image Processing: An International Journal (SIPIJ) Vol.9, No.6, December 2018

are sensitive to noise. On the other hand, contour-based corner detectors are more stable and robust than intensity-based corner detectors [3]. But the most challenging part is that, the corners do not contain any significant information that can be used to build the interest region. Thus the region estimation process for these corner detectors are being done in the description stage.

Defining the size of the interest region is an important task as the image structure inside the region needs to be distinct to build a distinct descriptor. In our paper, we introduced a new local maxima-based interest region detection method around the detected corner location. The pixels within this region is then used to build a descriptor. We have used most popular SIFT descriptor [12] in this regard.

The rest of the paper is organised as follows. Section 2discusses some related works to estimate the regions in literature. Section 3 explains the proposed region estimation method. Section 4 presents the experimental results. Section 4.4 describes the use of proposed method in an image matching based application. Finally, Section 5 concludes the paper.

\section{RELATED WORKS}

In our work, we consider corner as the feature as it is more stable than others [3]. Among the two types of corner detectors, intensity-based method $[4,5]$ typically use scale-space representation process to calculate the region. Most of the corner detectors use several geometric parameters such as second moment matrix. Lindeberg [6] used The Laplacianand scale space determent of the Hessian to represent elliptical regions. Auto correlation matrices are used in Harris Affine and Laplace detectors [7] to detect regions. On theother hand contour-based corner detectors $[8,9,10]$ are morerobust and the interest region needs to be estimated in thedescription stage. Awrangjeb [11] used SIFT descriptor [12] to calculate the region within 3-pixels of the corner location.Awrangjeb [11] used CPDA [8] corner detector and considered the SIFT descriptors (detected by [12]) within the3-pixel neighbourhood around the corner locations. However, it lacks robustness as under different image transformations, the same SIFT descriptor might not detected forthe transformed image. For the same reason, there might be less possibilities to find the same corners for the originaland the transferred images. Sadat et el [13] used a scale-invariant estimation method which differentiate distinct contents around the corner location. They used a series of circular regions using the corner location as centre. They onlyselected the circular region which is different than the neighbouring circles based on the threshold. [5] used the edgesnear the detected corners for the region estimation to buildscale invariant descriptors. Later proposed Harris-Laplace[7] method performed better compared to that method. Many recent algorithms perform slightly better, such as Wash detector [2] and Boundary Preserving dense Local Regions (BPLR) [1]. Wewill propose a new method in section 3, which is more effective in estimating interest regions around a detected cornerfor building a scale invariant descriptor.

\section{Proposed Method}

As mentioned earlier, one of the most important stepsto describe local features is to estimate the interest regionaround the feature location to achieve the invariance againstdifferent image transformation. After the estimation, thepixels inside the interest region are used to build the descriptor. The descriptors, later, represent a feature.

In this section, we propose a new curvature-based interestregion detection method to detect corners using contour-based corner detectors. Almost all of the contour-based corner detectors first extract the edges of an image using anyedge detectors such as canny [14]. The basic idea of estimating interest region is to select the curvature maxima ofthe edges. The estimated region needs to be defined wellenough to represent that corner so that in the transformedimage, the 
corresponding corner can be estimated with thesame content. To do so, first, we detect the corner locations of an image. Now we estimate the curvature value to find the corner locations. During estimating the curvaturevalues we also calculate the maxima of the curvatures foreach corners of each edges. For each corner we calculatethe distance of the nearest maxima. If the distance fromthe corner location to a curvature maxima is larger thana predefined distance $D_{i}$, we will discard the maxima. Inour method we selected Di as 80. Now for the rest of thecandidate maxima, we applied a simple refinement process.In this refinement method, for a particular corner location,we consider all the maxima found after extracting edges. Ifthe distance of two adjacent maxima is lower than 3 pixel,we compute the average of two distances from the cornerlocation.

The pseudo-code for selecting radii of our proposed methodis presented in Figure 1. We will use these radii as thecircular regions to build descriptors which will later represent the corner.

Table 1. Algorithm for the proposed model

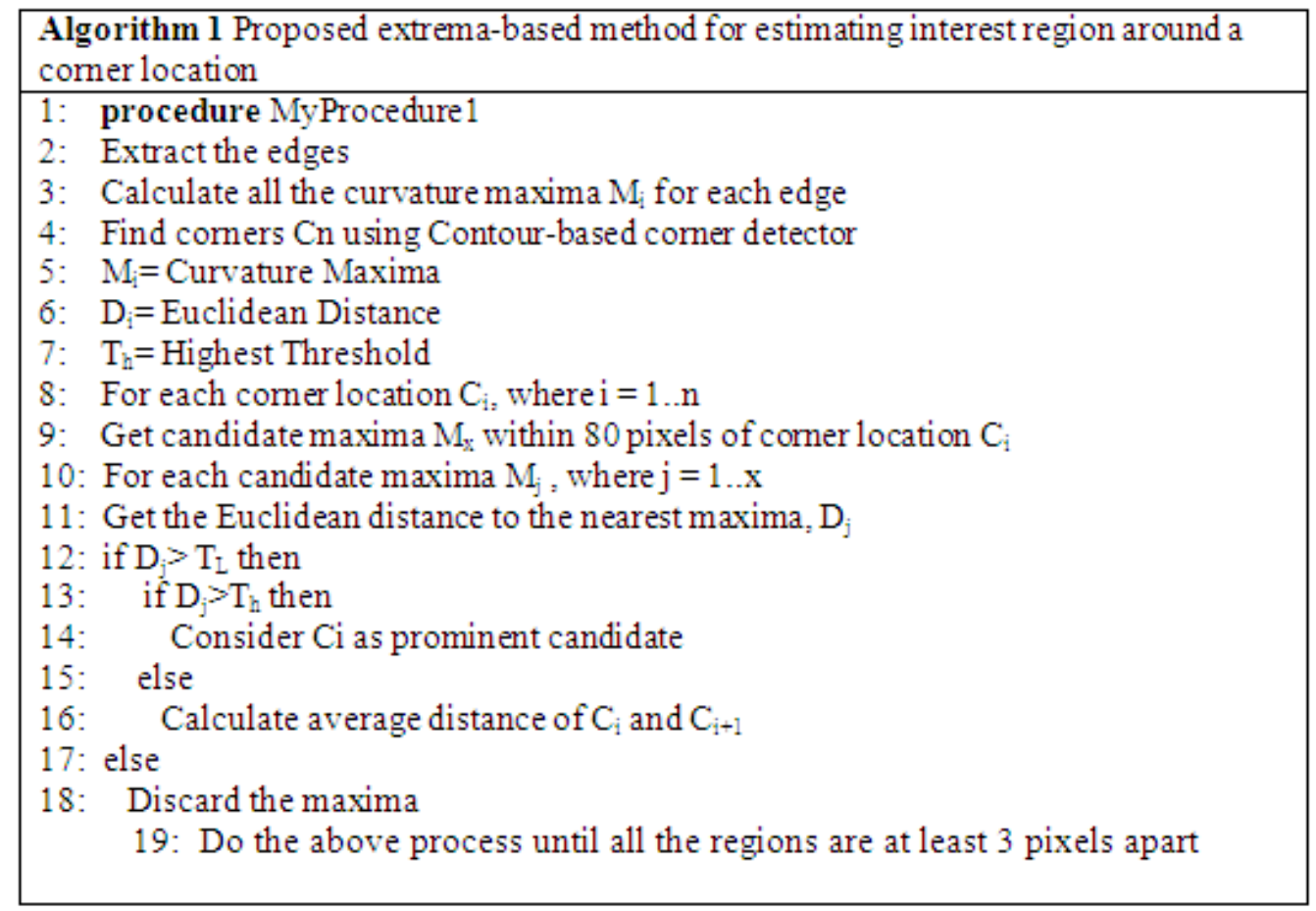

Figure 1 shows the corners detected by MSCAD corner detector [10] as the centre of the circular regions. MSCAD [10]is a recent contour-based corner detector, which has achieved better repeatability and comparable localization errors under different transformations compared to other state-of-artscontour-based corner detectors. We will calculate the circular region around a particular corner location using the pseudocode mentioned above. 


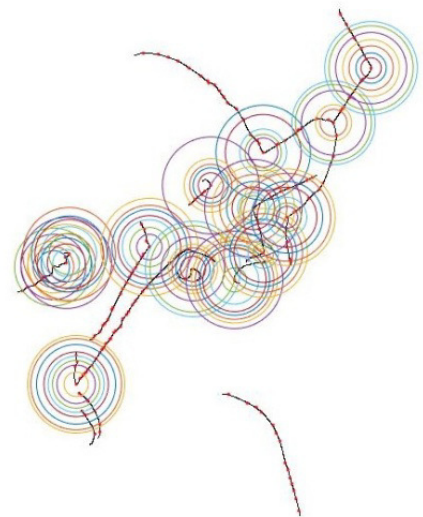

(a)

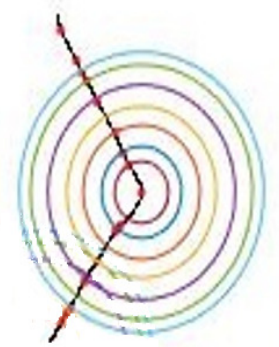

(b)

Figure 1. Interest region detection using edge extrema.(a)Corners detected by corner detector, (b) Selected region of a corner location

\section{Performance Study}

In this section, we compare our proposed method withHarris-Laplace[7],Awrangjeb'sMethod [11]and the LoG method[18]. We use MSCAD[10], CCR[16], CPDA [8] corner detectionmethods in this regard to observe the performances ofthe interest region detection process. We have used SIFT[12] as feature descriptor for all the methods. All the experiments were run on MATLAB 2018a on a Windows 10 (64bit)machine with an Intel Core i5-3470 processor and 8GB of RAM.

\subsection{Test Database}

We have used an image dataset of 23 different types ofgrey scale images to evaluate the performance of the cornerdetectors using different edge detectors. Different transformations have been applied to these base 23 images. Table 2 tabulates the geometric transformations applied to theseimages to obtain more than 8000 transformed test images.

Table 2. Image Transformations applied on 23 base images

\begin{tabular}{|l|l|l|}
\hline Transformations & Transformation Factors & Number of Images \\
\hline Scaling & $\begin{array}{l}\text { Scale factors } \mathrm{s}_{\mathrm{x}}=\mathrm{s}_{\mathrm{y}} \mathrm{in}[0.5,2.0] \text { at } 0.1 \text { apart, } \\
\text { excluding } 1.0\end{array}$ & 345 \\
\hline Shearing & $\begin{array}{l}\text { Shear factors } \mathrm{sh}_{\mathrm{z}} \text { and } \mathrm{s}_{\mathrm{y}} \text { in }[0,0.012] \text { at } \\
0.002 \text { apart }\end{array}$ & 1081 \\
\hline Rotation & $\begin{array}{l}18 \text { different anglesof range }-90^{\circ} \text { to }+90^{\circ} \text { at } \\
10^{\circ} \text { apart }\end{array}$ & 437 \\
\hline Rotation-Scale & $\begin{array}{l}\text { in }[-30,+30] \text { at } 10^{\circ} \text { apart, followedby } \\
\text { uniform and non-uniform scale factors } \mathrm{s}_{\mathrm{x}} \\
\text { and } \mathrm{s}_{\mathrm{y}} \text { in }[0.8,1.2] \text { at } 0.1 \text { apart }\end{array}$ & 4025 \\
\hline Non-uniform Scale & $\begin{array}{l}\text { Scale factors } \mathrm{s}_{\mathrm{x}} \text { in }[0.7,1.3] \text { and } \mathrm{s}_{\mathrm{y}} \text { in }[0.5, \\
1.5] \text { at } 0.1 \text { apart. }\end{array}$ & 1772 \\
\hline Gaussian Noise & $\begin{array}{l}\text { Gaussian }(\mathrm{G}) \text { noiseat } 10 \text { variances in } \\
{[0.005,0.05] \text { at } 0.005 \text { apart. }}\end{array}$ & 230 \\
\hline
\end{tabular}




\subsection{Evaluation metrics}

To evaluate the performance against different transformations, we use commonly used precision and recall measure[1]. Precision and recall represents the number of correctmatches with respect to total number of matches and number of correct matches with respect to the total number ofcorrespondence respectively. Thus, precision and recall canbe measured by the equation 1 and 2.

$$
\begin{aligned}
& \text { Precision }=\frac{\text { Number of correct matches }}{\text { Total number of matches }} \\
& \text { Recall }=\frac{\text { Number of correct matches }}{\text { Total number of correspondences }}
\end{aligned}
$$

\subsection{Experimental results}

In our experiment, we empirically define the number of bins in the scale-histogram and the threshold(th) for measuring the dissimilarities between two circular regions as 40 and 0:32 respectively. To compare the descriptors of two images, we use the commonly used nearestneighbourhood strategy. Our proposed method and the LoG method may have the possibility to have multiple interest regions for a single corner location. Thus the matching descriptors might not belong to a single corner location. To overcome the problem, we have applied a simple refinement process by which we can easily filter out the false matches, keeping the true matches. To do this, we consider only the matches which correspond to the highest number of matching relations between two corner locations of the two input images.

We evaluate the performance of our proposed method with other existing methods with precision and recall measure using the equations 1 and 2 as mentioned in the previous section. Figure 2 to 7 shows the performance comparison of all the methods using precision vs recall graph. From those results, we can easily observe that our proposed method along with the MSCAD [10] corner detector performs consistently better compared to other methods under all the image transformations. The result also shows that, for JPEG compression, Log approach is almost similar to our proposed method as there is no geometric transformation. Although the precision of Harris-Laplace is better in a few cases when the recall is higher, the recall of the proposed method is always higher than Harris-Laplace. Compared to other methods, Awrangjeb's method and the LoG method do not show consistent performance. Our proposed method is better than these two methods in terms of both precision and recall. Awrangjeb's method has better precision than Harris-Laplace only when the recall is lower. 
Signal \& Image Processing: An International Journal (SIPIJ) Vol.9, No.6, December 2018

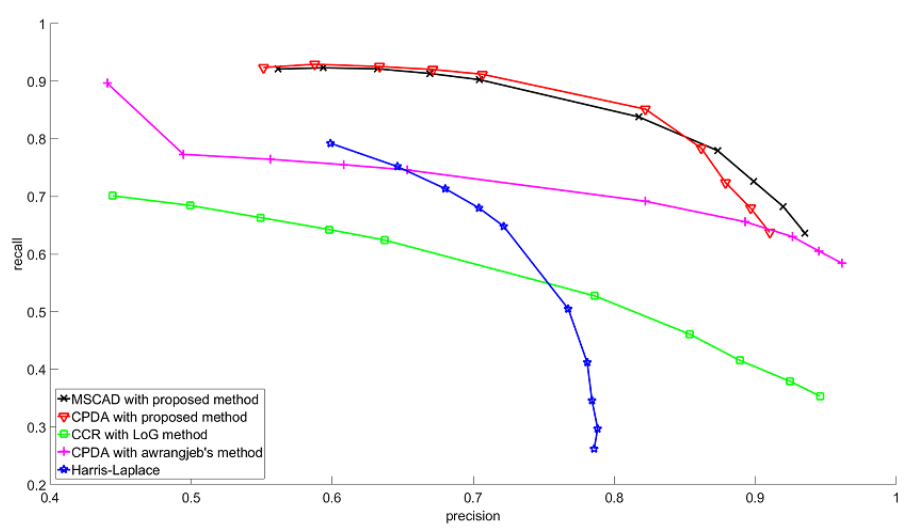

Figure 2. Performance evaluation of different methods under scale transformations

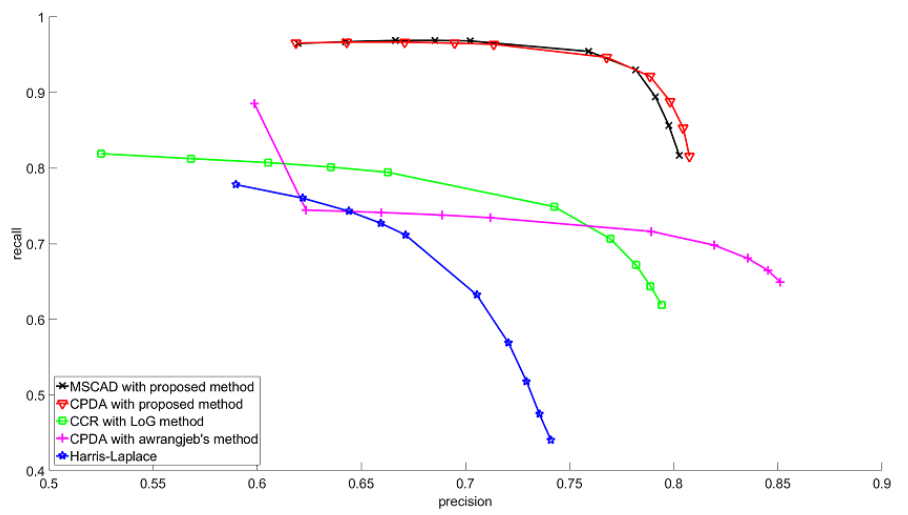

Figure 3. Performance evaluation of different methods under shear transformations

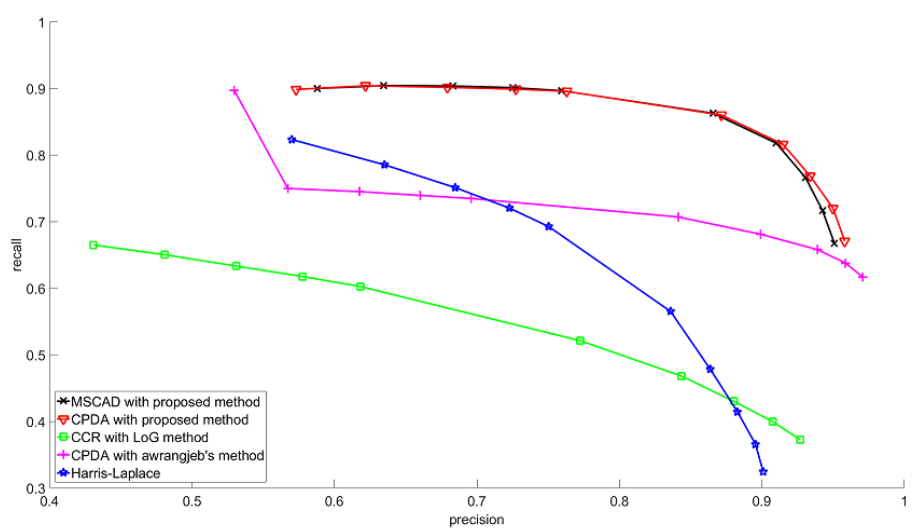

Figure 4. Performance evaluation of different methods under rotation transformations 
Signal \& Image Processing: An International Journal (SIPIJ) Vol.9, No.6, December 2018

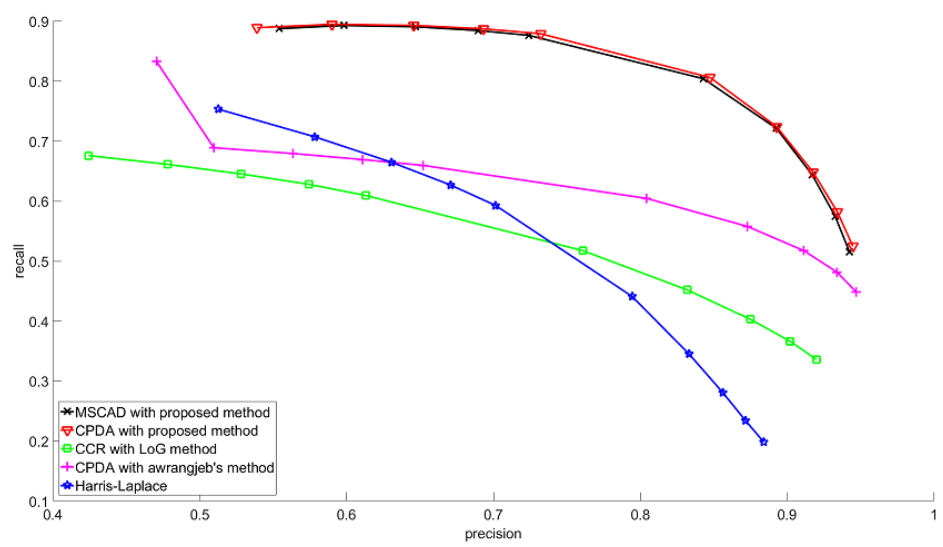

Figure 5. Performance evaluation of different methods under rotation and scale transformations

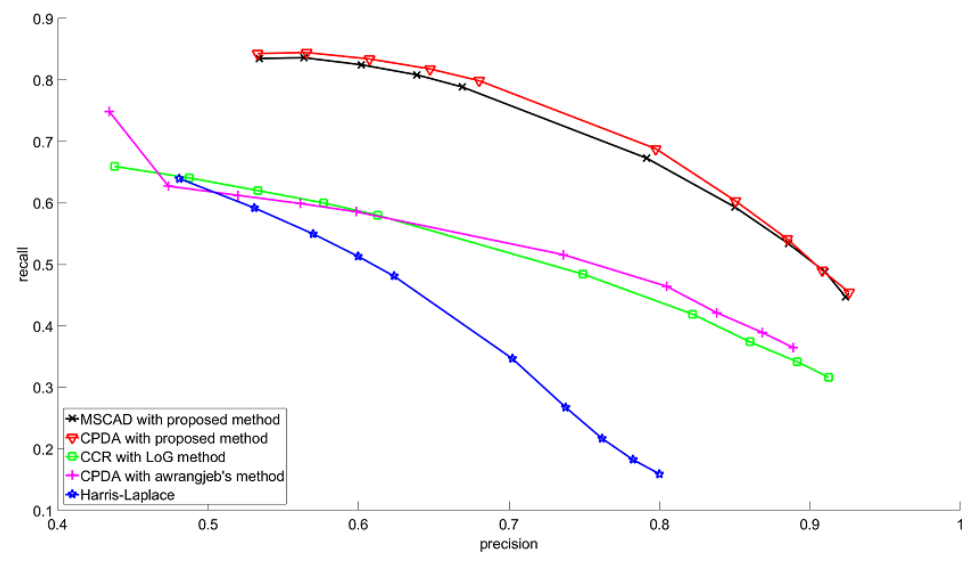

Figure 6. Performance evaluation of different methods under non-uniform transformations

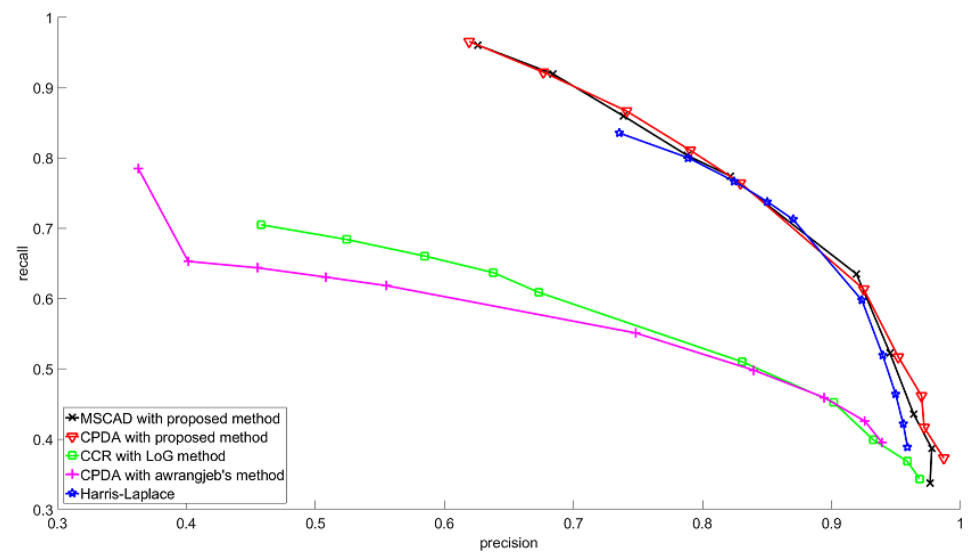

Figure 7. Performance evaluation of different methods under Gaussian transformations 


\subsection{Application}

In this section, we present an application of image matching with the proposed interest region estimation method. Now in order to match the corner pairs between two images, we detect the corner locations from the images and then we describe each corner using a SIFT descriptor. To represent a feature, we determine the interest region (Section 3) around the corner locations. Next, an orientation histogram is created from the gradient magnitude and gradient orientation of all the pixels within the interest region. The peak in the histogram corresponds to the dominant orientation of that feature location. To make the feature descriptor rotational invariant, the interest region is rotated along the dominant orientation as proposed by[15], [12].

To build the descriptor, we start with computing a circular SIFT frame proposed by [12] as a vector with four parameters, which contains the circle centre with an orientation and scale. Next, the descriptor for each SIFT frame is built based on the pixel values of the oriented interest region. In the standard SIFT descriptor representation, each descriptor is a 128-dimensional feature vector [12]. To match the feature descriptors, first nearest neighbour of descriptors is used. To eliminate any mismatches, we use Lowe's second nearest neighbour test. The main task is to figure out distinctive matches by a threshold on the ratio of first to second nearest neighbour distances. Finally, we represent all of the descriptors of an image and assess the similarity between two images via sparse matching.

Now we build SIFT descriptors for the corner locations from the reference image and the test images. Next, we find the matched descriptors and verify the matches based on the geometric transformation between the images. Please note that, the geometric transformation for the images are given with the image dataset. We are using CCR [16] and MSCAD [10] detectors in this experiment. The examples of matching corners after verifying the matches is shown in Figure 8 for both MSCAD and CCR detectors.

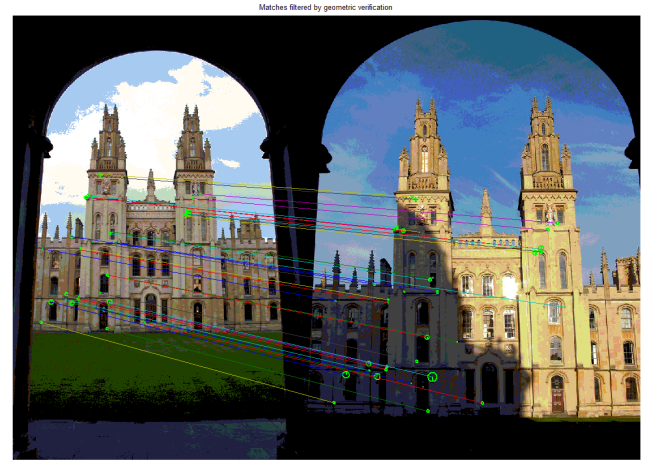

(a) Using MSCAD detector

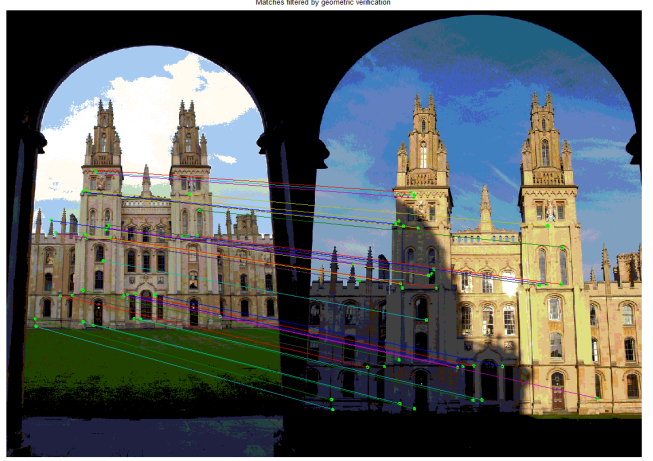

(b) Using CCR detector

Figure 8. Matching after Geometric Verifications

Table 3 shows average number of matches and average number of true matches among the base image and test images. MSCAD corner detector performs better than CCR for finding true matches. 
Signal \& Image Processing: An International Journal (SIPIJ) Vol.9, No.6, December 2018

Table 3. Feature Matching after Geometric Verifications

\begin{tabular}{|l|l|l|}
\hline Method & Descriptor Match & True Match \\
\hline MSCAD & 116.69 & 67.15 \\
\hline CCR & 112 & 61.56 \\
\hline
\end{tabular}

\section{CONCLUSION}

This paper proposed a new algorithm to calculate the interest region around the corner location of an image using the curvature maxima. Our proposed method represent the corners using contourbased corner detectors and local feature descriptors without using the scale-space representation. Using our proposed method, corners can be tracked and matched nearly accurate in the transformed images. The experimental results show that our proposed method performs better in compared to other methods in terms of precision and recall values, which also indicates that the descriptors built using the interest region are invariant to different image transformations.

\section{REFERENCES}

[1] J. Kim and K. Grauman. Boundary preserving dense local regions. IEEE transactions on pattern analysis and machine intelligence, 37(5):931-943, 2015.

[2] C. Varytimidis, K. Rapantzikos, and Y. Avrithis. Wash: Weighted a-shapes forlocal feature detection. In Computer Vision-ECCV 2012, pages 788-801. Springer,2012

[3] F. Mokhtarian and R. Suomela, LRobust image cornerdetection through curvature scale space," IEEE Trans.Pattern Anal. Mach. Intell., vol. 20, no. 12, pp.1376\{1381, 1998.

[4] C. Harris and M. Stephens, \A combined corner andedge detection," pp. 147\{151, 1988.

[5] T. Tuytelaars and L. Van Gool, IMatching widelyseparated views based on affine invariant regions," Int.J. Comput. Vision, vol. 59, no. 1, pp. 61\{85, Aug.2004. [Online]. Available: http://dx.doi.org/10.1023/B:VISI.0000020671.28016.e8

[6] T. Lindeberg, IScale selection for differentialoperators," in Scale-Space Theory in Computer Vision.Springer, 1994, pp. $317\{348$.

[7] K. Mikolajczyk and C. Schmid, IScale \& affineinvariant interest point detectors," Int. J. Comput.Vision, vol. 60, no. 1, pp. 63\{86, Oct. 2004.

[8] M. Awrangjeb and L. Guojun, \Robust image cornerdetection based on the chord-to-point distanceaccumulation technique," Multimedia, IEEETransactions on, vol. 10, no. 6, pp. 1059\{1072, 2008.

[9] S. W. T. R. M. Najmus Sadat and G. Lu, \Aneffective and efficient contour-based corner detectorusing simple triangular theory," pp. 37\{42, 2011.

[10] N. Afrin, N. Mohammed, and W. Lai, \An effectivemulti-chord corner detection technique," in DigitalImage Computing: Techniques and Applications(DICTA), 2016 International Conference on. IEEE,2016, pp. $1\{8$.

[11] M. Awrangjeb and G. Lu, ITechniques for efficientand effective transformed image identification," J. Vis.Comun. Image Represent. vol. 20, no. 8, pp. 511\{520,Nov. 2009. [Online]. Available:http://dx.doi.org/10.1016/j.jvcir.2009.07.004 
[12] D. G. Lowe, Distinctive image features fromscale-invariant keypoints," Int. J. Comput. Vision, vol. 60, no. 2, pp. $91\{110$, Nov. 2004.

[13] R. Sadat, S. W. Teng, and G. Lu, \An effectivemethod of estimating scale-invariant interest regionfor representing corner features," in Image and VisionComputing New Zealand (IVCNZ), 2012 27thInternational Conference of, nov. 2012.

[14] J. Canny, \A computational approach to edgedetection," Pattern Analysis and Machine Intelligence,IEEE Transactions on, vol. PAMI-8, no. 6, pp. 679\{698, nov. 1986.

[15] D. Lowe, lObject recognition from localscale-invariant features," in Computer Vision, 1999. The Proceedings of the Seventh IEEE InternationalConference on, vol. 2, 1999, pp. 1150 \{1157 vol.2.

[16] S. W. T. R. M. Najmus Sadat and G. Lu, \Aneffective and efficient contour-based corner detectorusing simple triangular theory," pp. 37\{42, 2011.

[17] K. Mikolajczyk and C. Schmid, \A performanceevaluation of local descriptors," Pattern Analysis andMachine Intelligence, IEEE Transactions on, vol. 27,no. 10, pp. 1615\{1630, Oct. 2005.

[18] T. Lindeberg, IFeature detection with automatic scaleselection," Int. J. Comput. Vision, vol. 30, no. 2, pp.79\{116, Nov. 1998. [Online]. Available:http://dx.doi.org/10.1023/A:1008045108935

\section{Authors}

Naurin Afrin is currently a $\mathrm{PhD}$ student of Computer Science and Engineering department.Her research is focused on image processing, computer vision and pattern recognition.

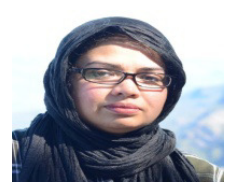

Wei Lai is currently a Senior Lecturer at Swinburne University of Technology, Australia in department of Computer Science and Software Engineering. His research interest includes image processing and information visualisation.

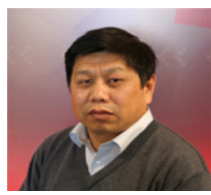

